TUW-99-02

Vers. 2.1

\title{
Absolute conservation law for black holes
}

\author{
D. Grumiller* and W. Kummer! \\ Institut für Theoretische Physik \\ Technische Universität Wien \\ Wiedner Hauptstr. 8-10, A-1040 Wien \\ Austria
}

\begin{abstract}
In all $2 \mathrm{~d}$ theories of gravity a conservation law connects the (spacetime dependent) mass aspect function at all times and all radii with an integral of the matter fields. It depends on an arbitrary constant which may be interpreted as determining the initial value together with the initial values for the matter field. We discuss this for spherically reduced Einstein-gravity in a diagonal metric and in a BondiSachs metric using the first order formulation of spherically reduced gravity, which allows easy and direct fixations of any type of gauge. The relation of our conserved quantity to the ADM and Bondi mass is investigated. Further possible applications (ideal fluid, black holes in higher dimensions or AdS spacetimes etc.) are straightforward generalizations.
\end{abstract}

PACS numbers: 0470Bw, 0425Dm, 1130-j

${ }^{*}$ e-mail: grumil@hep.itp.tuwien.ac.at

$\dagger$ e-mail: wkummer@tph.tuwien.ac.at 


\section{Introduction}

The treatment of two-dimensional models of gravity is simpler and more transparent in terms of a light-cone gauge for the Cartan variables, or, equivalently, in Eddington-Finkelstein (EF) gauge for the 2d metric [1], especially when these approaches are combined with the first order formulation

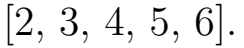

Of course using traditional approaches - e.g. performing the calculations in conformal gauge [7, 8] - one must be able to obtain the same results. In practice, however, the resulting equations of motion are rather complicated. Therefore, it is not surprising that also a complete discussion of the global solution of such models has turned out to be easier in the EF gauge [9] than in the corresponding previous studies of the conformal gauge [10 for the model of Katanaev and Volovich [11]. Based upon the use of the EF metric in a first order formulation also substantial progress has been achieved in the meantime regarding the quantum theory of such models 12 .

The purpose of our present note is to draw attention to one particular aspect of the first order approach to spherically reduced gravity, namely the existence of an absolute (space and time) conservation law connecting the effective (space-time) dependent mass aspect function with the interacting matter fields [5, 13. This conservation law generalizes some previously known special cases, like e.g. the case without matter 14 and the one with nondynamical matter 15. The differential equations describing spherically symmetric Einstein gravity interacting with matter are known for a long time. Many recent extensive numerical simulations of black hole formation [16, 17] have brought important insight regarding critical phenomena related to a collapsing gravitating system. However, deriving the differential equations directly from the $4 \mathrm{~d}$ Einstein equation obscures the appearance of the conservation law mentioned above. This is probably the reason why - to the best of our knowledge - it has not been noted and employed in this context before. The underlying Noether symmetry - which will not be the issue of this paper - has been investigated in 13.

For the reader who is not too familiar with the description of spherically reduced Einstein gravity by an equivalent first order action with torsion in terms of Cartan variables we recapitulate the action and the equations of motion in Section II.

In Section III we fix the gauge so as to produce a diagonal $2 \mathrm{~d}$ metric. Then the equations used in some recent $\mathrm{BH}$ simulations [18] are reproduced. 
For easier comparison our notations have been adapted to the ones in that reference. We then derive the conservation law in this gauge.

Section IV is devoted to a EF gauge which, in our formulation, provides a very similar system of equations. This gauge, which in the non-static case is also known as (ingoing) Bondi-Sachs (BS) gauge - c.f. e.g. [19], seems to be especially adequate for computations involving both sides of the horizon, because it avoids the coordinate singularity, which could possibly present problems in the diagonal gauge. Our system of equations in that gauge resembles closely the one in the diagonal gauge and seems to be remarkably simple in comparison with the one used in earlier numerical work with BS gauge fixation [20]. Of course the mathematical content of both approaches is identical. Therefore, it must be possible to eliminate the extrinsic curvature appearing in [20]. An explicit example of such an elimination in a derivation of the spherically reduced action equation (2) below has been given in the appendix of the second reference of [21]. Finally a simple relationship between the integrated mass-aspect function, the conservation law, the Bondi mass $\left(\right.$ at $\mathcal{I}^{-}$) and the ADM mass is established.

In the conclusions we list further applications where the same arguments can be applied with equal ease.

\section{First order formulation of spherically re- duced gravity}

In terms of the spherically symmetric ansatz for the line element $\left(g_{\mu \nu}=\right.$ $g_{\mu \nu}(x)=g_{\mu \nu}(t, r)$ with the dilaton field defined by $\left.\phi(x)\right)$

$$
(d s)^{2}=g_{\mu \nu} d x^{\mu} d x^{\nu}-\frac{4 e^{-2 \phi}}{\lambda^{2}}(d \Omega)^{2}
$$

after integrating out the angular variables $\int d^{2} \Omega$ of $S^{2}$, the Einstein-Hilbert Lagrangian in 4 dimensions with Newton constant $G=1$ reduces to [21] a dilaton theory in $d=2$ :

$$
L_{d i l}^{(g)}=\int d^{2} x \mathcal{L}_{d i l}^{(g)}, \mathcal{L}_{d i l}^{(g)}=\sqrt{-g} e^{-2 \phi}\left(R+2(\nabla \phi)^{2}-\frac{\lambda^{2}}{2} e^{2 \phi}\right)
$$

In a similar way the Lagrangian describing minimally coupled scalars $S$ in $d=4$ becomes

$$
\mathcal{L}^{(m)}=8 \pi \sqrt{-g} e^{-2 \phi}(\nabla S)^{2}
$$


Expressions like $(\nabla S)^{2}=g^{\mu \nu}\left(\partial_{\mu} S\right)\left(\partial_{\nu} S\right)$ involve the 2 d metric in (1). Introducing a new variable

$$
X=e^{-2 \phi}
$$

for the dilaton field, the Lagrangian (3) can be verified to be equivalent to the first order action of a $2 d$ theory with nonvanishing torsion] [22]

$$
\begin{aligned}
\mathcal{L}^{(g)} & =-\left(X^{+} D \wedge e^{-}+X^{-} D \wedge e^{+}+X d \omega-e^{-} \wedge e^{+} \mathcal{V}\right) \\
\text { with } \mathcal{V} & =V(X)+X^{+} X^{-} U(X)
\end{aligned}
$$

where $U(X)=-\frac{1}{2 X}$ and $V(X)=-\frac{\lambda^{2}}{4}$ for spherically reduced gravity

with the covariant derivative $D \wedge e^{ \pm}=d \wedge e^{ \pm} \pm \omega \wedge e^{ \pm}$.

Equation (5) is expressed in terms of Cartan variables, the zweibeine $e^{a}$ and the spin connection $\omega^{a b}=\omega \varepsilon^{a b}$. $\varepsilon^{\mu \nu}$ represents the antisymmetric LeviCivitá symbol $\left(\varepsilon^{01}=-\varepsilon_{01}=1\right)$. In the local Lorentz light-cone coordinates $(a=(-,+))$

$$
\eta^{a b}=\eta_{a b}=\left(\begin{array}{ll}
0 & 1 \\
1 & 0
\end{array}\right)
$$

the relation to the $2 \mathrm{~d}$ metric is

$$
\begin{aligned}
g_{\mu \nu} & =e_{\mu}^{a} e_{\nu}^{b} \eta_{a b} \\
\sqrt{-g} & =(e)=e_{0}^{-} e_{1}^{+}-e_{1}^{-} e_{0}^{+},
\end{aligned}
$$

and the $2 \mathrm{~d}$ curvature scalar becomes

$$
\sqrt{-g} R=-2 \varepsilon^{\mu \nu} \partial_{\nu} \widetilde{\omega}_{\mu}
$$

where $\widetilde{\omega}_{\mu}$ denotes the spin-connection for vanishing torsion (i.e. when the term involving $X^{ \pm}$in (5) were absent). The equivalence of (2) with (5), using (8) and (4) can be checked easily. The algebraic equations of motion for $\omega_{0}$ and $\omega_{1}$ from the variation with respect to $X^{ \pm}$are reinserted into (5), then $X^{ \pm}$can be eliminated in a similar manner.

\footnotetext{
${ }^{1}$ In (5) as well as in all formulae derived from it below an overall factor 2 has been dropped.
} 
Now also (3) should be expressed by Cartan variables. From the identity

$$
\sqrt{-g} g^{\alpha \beta}=-\frac{\varepsilon^{\alpha \rho} \varepsilon^{\beta \sigma}}{(e)}\left(e_{\rho}^{+} e_{\sigma}^{-}+e_{\rho}^{-} e_{\sigma}^{+}\right)
$$

in terms of the abbreviations

$$
S^{ \pm}=\varepsilon^{\alpha \rho} e_{\rho}^{ \pm} \partial_{\alpha} S
$$

equation (3) becomes:

$$
\mathcal{L}^{(m)}=-\frac{8 \pi X}{(e)} S^{+} S^{-}
$$

The equations of motion (e.o.m.) for the sum of the geometric Lagrangian (5) and the matter Lagrangian (12) are

$$
\begin{aligned}
d X+X^{-} e^{+}-X^{+} e^{-} & =0 \\
d X^{ \pm} \pm \omega X^{ \pm} \mp e^{ \pm}\left(V(X)+X^{+} X^{-} U(X)\right)+M^{ \pm} & =0 \\
d \omega-e^{-} \wedge e^{+}\left(V^{\prime}(X)+X^{+} X^{-} U^{\prime}(X)\right)-\frac{\delta \mathcal{L}^{(m)}}{\delta X} & =0 \\
(d \pm \omega) \wedge e^{ \pm}-e^{-} \wedge e^{+} X^{ \pm} U(X) & =0 \\
d\left(\frac{F(X)}{(e)}\left(e^{+} S^{-}+e^{-} S^{+}\right)\right) & =0
\end{aligned}
$$

with

$$
M^{ \pm}=-\frac{\delta \mathcal{L}^{(m)}}{\delta e^{ \pm}}
$$

using the left-derivative.

In the system of equations (13)-(17) the $2 \mathrm{~d}$ gauge fixing is still free. This is one of the advantages of the approach starting from a spherically reduced action. The gauge symmetry of (5) and (12) consists of two diffeomorphisms and one local Lorentz transformation $\mathcal{G}=\operatorname{Diff}_{2} \times S O(1,1)$. Thus the equations are not independent.

In this first order formulation the absolute conservation law is obtained easily [5]. Combining

$$
\begin{array}{r}
X^{+} \times\left[e q \cdot(14)^{-}\right]+X^{-} \times\left[e q \cdot(114)^{+}\right] \\
+\left(V(X)+X^{+} X^{-} U(X)\right) \times[e q .(13)]
\end{array}
$$


where eq. $(\sqrt{14})^{ \pm}$is the e.o.m. from $\delta e^{\mp}$, we obtain with an integrating factor

$$
I(X)=\exp \left[\int^{X} U\left(X^{\prime}\right) d X^{\prime}\right]
$$

(which simplifies to $I(X)=X^{-\frac{1}{2}}$ in the case of spherically reduced gravity)

$$
d \mathcal{C}^{(g)}+W^{(m)}=0
$$

with

$$
\begin{aligned}
\mathcal{C}^{(g)} & =I(X) X^{+} X^{-}+\int^{X} V\left(X^{\prime}\right) I\left(X^{\prime}\right) d X^{\prime} \\
W^{(m)} & =I(X)\left(M^{+} X^{-}+M^{-} X^{+}\right) .
\end{aligned}
$$

The integrability condition $d W^{(m)}=0$ can be verified to be a consequence of the e.o.m. (13)-(16) [13], but it also follows trivially from the definition (20). Therefore, with $W^{(m)}=d \mathcal{C}^{(m)}$ the conservation law reads $d\left(\mathcal{C}^{(g)}+\mathcal{C}^{(m)}\right)=0$.

For the case of spherically reduced gravity we obtain in components

$$
\partial_{\mu} \mathcal{C}^{(g)}+W_{\mu}^{(m)}=0
$$

where

$$
\begin{aligned}
\mathcal{C}^{(g)} & =\frac{X^{+} X^{-}}{\sqrt{X}}-\frac{\lambda^{2}}{2} \sqrt{X} \\
W_{\mu}^{(m)} & =\frac{8 \pi \sqrt{X}}{(e)^{2}}\left[(e)\left(\partial_{\mu} S\right)\left(S^{-} X^{+}+S^{+} X^{-}\right)-S^{+} S^{-} \partial_{\mu} X\right]
\end{aligned}
$$

In the absence of matter $\left(W^{(m)}=0\right)$ the quantity $\mathcal{C}^{(g)}<0$ is proportional to the mass of the black hole; in the presence of matter it becomes the mass aspect function (see below).

\section{Diagonal gauge}

The choice of the gauge for $g_{\mu \nu}$ in (11) with equation (4) (cf. ref. [18]; we use as in (1) the opposite convention for the sign of $\left.(d s)^{2}\right)$

$$
\begin{aligned}
g_{\mu \nu} & =\left(\begin{array}{cc}
\alpha^{2}(t, r) & 0 \\
0 & -a^{2}(t, r)
\end{array}\right) \\
X & =\frac{\lambda^{2} r^{2}}{4}
\end{aligned}
$$


for the zweibeine $e_{\mu}^{ \pm}$yields the conditions

$$
e_{0}^{+}=e_{0}^{-}=\frac{\alpha}{\sqrt{2}}, \quad e_{1}^{+}=-e_{1}^{-}=\frac{a}{\sqrt{2}} .
$$

In the gauge (26), (27) equation (17) is simply solved by

$$
X^{+}=X^{-}=-\frac{\lambda^{2} r}{2 \sqrt{2} a} .
$$

Taking the sum and the difference of equations (16) the algebraic relations for the spinor connection $\omega_{\mu}$ are obtained:

$$
\omega_{0}=\frac{\partial_{1}(\alpha r)}{a r}, \quad \omega_{1}=\frac{\partial_{0} a}{\alpha}
$$

For easier comparison with the equations of motion in ref. [18 the same abbreviations

$$
\partial_{0} S=\frac{\partial S}{\partial t}=\frac{\alpha}{a} \Pi, \quad \partial_{1} S=\frac{\partial S}{\partial r}=\Phi
$$

are introduced. From (17) immediately the matter equations ((5) and (6) of ref. [18]) are reproduced:

$$
\begin{aligned}
\partial_{0} \Pi & =\frac{1}{r^{2}} \partial_{1}\left(r^{2} \frac{\alpha}{a} \Phi\right) \\
\partial_{0} \Phi & =\partial_{1}\left(\frac{\alpha}{a} \Pi\right)
\end{aligned}
$$

The "Hamiltonian constraint" (equation (8) of ref. [18]) follows from the sum of the equations (14) for the component $\mu=1$ :

$$
\partial_{1} \ln a+\frac{a^{2}-1}{2 r}-2 \pi r\left(\Phi^{2}+\Pi^{2}\right)=0
$$

Comparing this with the difference of the zero components of the same two equations results in the "slicing condition" of ref. 18

$$
\partial_{1} \ln \left(\frac{\alpha}{a}\right)+\frac{1-a^{2}}{r}=0
$$


All other combinations of equations (14)-(15) are found to yield dependent equations except the zero component of the sum of equations (14)

$$
\partial_{0} a=4 \pi r \alpha \Phi \Pi
$$

which, as will be seen below, in a certain sense contains further information.

Having verified that the basic equations (32)-(35) are identical to the ones following from the $4 \mathrm{~d}$ Einstein equations 2 in we turn to the absolute conservation law (23). In the gauge (26), (27) its geometric part becomes

$$
\mathcal{C}^{(g)}=\frac{\lambda^{3} r}{4 a^{2}}\left(1-a^{2}\right)
$$

which in terms of a variable mass parameter $m(t, r)$, the so-called mass aspect function 18

$$
a^{-2}=1-\frac{2 m}{r}
$$

yields

$$
\mathcal{C}^{(g)}=-\frac{\lambda^{3}}{2} m
$$

i.e. in the absence of matter $\mathcal{C}^{(g)}$ is proportional to the mass of the (Schwarzschild) black hole.

The matter contribution to (23) in the present gauge with (31) becomes

$$
\begin{aligned}
& \partial_{0} \mathcal{C}^{(m)}=-\frac{\lambda^{3}}{2} \rho^{2}\left(2 \frac{\alpha}{a} \Pi \Phi\right), \\
& \partial_{1} \mathcal{C}^{(m)}=-\frac{\lambda^{3}}{2} \rho^{2}\left(\Phi^{2}+\Pi^{2}\right) .
\end{aligned}
$$

With (39) and the abbreviations [18

$$
\mathcal{X}=\rho \Phi, \quad \mathcal{Y}=\rho \Pi, \quad \rho=\frac{\sqrt{2 \pi} r}{a}
$$

\footnotetext{
${ }^{2}$ Although seemingly obvious at first glance this result is by no means trivial. E.g. for the more general ansatz of a "warped" metric in Einstein relativity [23] the complete set of e.o.m. cannot be derived from the reduced action. Also the treatment of quantum effects like Hawking radiation cannot be carried over straightforwardly to the spherically reduced case [24].
} 
the conservation law (23) can be brought into a very simple form:

$$
\begin{aligned}
\partial_{0} m & =2 \frac{\alpha}{a} \mathcal{X} \mathcal{Y}=A(t, r) \\
\partial_{1} m & =\mathcal{X}^{2}+\mathcal{Y}^{2}=B(t, r)
\end{aligned}
$$

Clearly in the present gauge these equations are nothing else but the equations of motion (34) and (36). Therefore in this particular case the whole formalism leading to (23) seems to be superfluous. However, as emphasized already above, the power of that approach becomes evident in other gauges, e.g. when the dilaton field $X=X(t, r)$ is not included among the gauge fixed quantities.

The integral of (43) and (44) can be written in two ways. Starting with the time integral of (43) and then using (44) with the integrability condition for the r.h.s. of these two equations yields

$$
m\left(t, r ; t_{0}, r_{0}\right)=\int_{t_{0}}^{t} d t^{\prime} A\left(t^{\prime}, r\right)+\int_{r_{0}}^{r} d r^{\prime} B\left(t_{0}, r^{\prime}\right)+m_{0}
$$

which determines $m$ for arbitrary $t, r$ from boundary values $r_{0}$ and $t_{0}$ and from the dynamical evolution of the scalar fields. The overall conserved value is represented by the constant $m_{0}$ which labels a certain solution much like the total energy in an ordinary conservative mechanical system. An equivalent formulation of the integral (45) is

$$
m\left(t, r ; t_{0}, r_{0}\right)=\int_{r_{0}}^{r} d r^{\prime} B\left(t, r^{\prime}\right)+\int_{t_{0}}^{t} d t^{\prime} A\left(t^{\prime}, r_{0}\right)+m_{0} .
$$

Equation (45) can be transformed directly into (46) by the use of the integrability condition for the r.h.s. of (43) and (44). It can be seen from (45) or (46) that equation (43), which is the same as (34), contains new information. Only the integrability condition involving its r.h.s. depends on the minimal set of equations. The new information is the one leading to the conserved quantity $m_{0}$.

It is instructive to extract the usual ADM mass [25] from (45). In the limit $r \rightarrow \infty$ for a system with an asymptotic Killing field $\frac{\partial}{\partial t}$ the ADM mass follows from (45)

$$
m_{A D M}=\int_{0}^{\infty} d r^{\prime} B\left(t_{0}, r^{\prime}\right)+m_{0}
$$


since $\frac{\partial m}{\partial t}=0$ requires vanishing $A(t, \infty)$. In (47) the lower limit of the integral has been changed with a simultaneous redefinition of $m_{0}$.

With the asymptotic spherical wave solutions in $A(u=t-r, v=t+r, a \rightarrow$ $1, \alpha \rightarrow 1)$

$$
S=\frac{1}{\sqrt{4 \pi} r}\left(f_{+}(u)+f_{-}(v)\right)
$$

from (31), (42), (43) and (44) the mass aspect function in the limit $r \rightarrow \infty$ becomes

$$
m_{B H}^{e f f}(t)=m\left(t, \infty ; t_{0}, 0\right)=m_{A D M}-\int_{t_{0}}^{t} d t^{\prime}\left[\left(f_{+}^{\prime}\right)^{2}-\left(f_{-}^{\prime}\right)^{2}\right],
$$

to be interpretated as the total effective mass of the (eventual) black hole. It consists of the usual ADM mass minus the difference of total outgoing and ingoing fluxes of matter at a certain time $t$. It is remarkable that a time dependent Bondi-like mass appears without having used the (for this purpose traditional) BS-gauge (see below). If in- or outgoing matter fluxes exist it is neccessary to use the effective black hole mass $m_{B H}^{\text {eff }}$ as a measure for black hole formation rather than $m_{A D M}$ alone [16].

\section{Bondi-Sachs gauge}

The advantage of the (ingoing) Eddington-Finkelstein gauge for the $2 \mathrm{~d}$ metric $g_{\mu \nu}$ in (1), defined either by $\left(x^{0}=v, x^{1}=r\right)$

$$
(d s)^{2}=(e)[h d v-2 d r] d v
$$

or by

$$
e_{1}^{+}=0, \quad e_{1}^{-}=-1
$$

and

$$
e_{0}^{+}=\operatorname{det} e=(e)=\sqrt{-g}, \quad e_{0}^{-}=\frac{h}{2}
$$

is its regular behavior at the event horizon. The Killing norm $(e) h$ for the Schwarzschild black hole has a simple zero. For this reason this gauge has 
been used also previously within studies of black hole formation (e.g. [20], [26]). In the presence of matter $(e)$ and $h$ are functions of $r$ and $v$ (ingoing $\mathrm{BS}$ gauge). In the first order approach in equations (13)-(17) now the gauge is fixed by (51) and (27).

Again (14) determines

$$
X^{+}=-\frac{\lambda^{2}}{2} r, \quad X^{-}=-\frac{h}{4(e)} \lambda^{2} r
$$

and as in the diagonal gauge the equations (16) define the spin connection:

$$
\omega_{0}=\frac{\partial_{1} h}{2}+\frac{h}{2} \frac{\partial_{1}(e)}{(e)}+\frac{h}{r}, \quad \omega_{1}=-\frac{1}{r}-\frac{\partial_{1}(e)}{(e)}
$$

From (14) for $\mu=1$ with (54) the BS-analogue of the "slicing condition" (35) of the diagonal gauge is obtained:

$$
\partial_{1} \ln h+\frac{1}{r}\left(1-\frac{(e)}{h}\right)=0
$$

It also does not involve derivatives $\partial_{0}$ and is even simpler than in the diagonal gauge. The "Hamiltonian constraint"

$$
\frac{r}{2} \partial_{1} \ln (e)-\hat{\mathcal{X}}^{2}=0
$$

is a consequence of (14) with $\mu=1$ and (54). In a similar way the analogue of equation (36)

$$
\partial_{0}\left(r \frac{h}{(e)}\right)-r \frac{h^{2}}{2} \frac{\partial_{1}(e)}{(e)^{2}}+\frac{4}{(e)} \hat{\mathcal{Y}}^{2}=0
$$

follows with the definitions

$$
\begin{aligned}
& \hat{\mathcal{X}}=\sqrt{2 \pi} r \partial_{1} S \\
& \hat{\mathcal{Y}}=\sqrt{2 \pi} r\left(\frac{h}{2} \partial_{1} S+\partial_{0} S\right)
\end{aligned}
$$

The e.o.m. (17) for the scalar field in terms of (58) and (59) become

$$
r \partial_{1} \hat{\mathcal{Y}}+\frac{h}{2} \hat{\mathcal{X}}=0
$$


and

$$
\left(\partial_{0}-\partial_{1} \frac{h}{2}\right) \frac{\hat{\mathcal{X}}}{r}=-\partial_{1}\left(\frac{\hat{\mathcal{Y}}}{r}\right)
$$

The geometric part of the conserved quantity (24) with (53) yields

$$
\mathcal{C}^{(g)}=\frac{\lambda^{3} r}{4}\left(\frac{h}{(e)}-1\right)
$$

which by comparison with the result (39) allows the introduction of a mass aspect function $\hat{m}(v, r)$. Evaluating $W_{0}$ and $W_{1}$ in (25) the absolute conservation law for $\hat{m}$ reads

$$
\begin{aligned}
& \partial_{0} \hat{m}=\frac{2}{(e)}\left(\hat{\mathcal{Y}}^{2}-\frac{h^{2}}{4} \hat{\mathcal{X}}^{2}\right)=\hat{A}(v, r), \\
& \partial_{1} \hat{m}=\frac{h}{(e)} \hat{\mathcal{X}}^{2}=\hat{B}(v, r),
\end{aligned}
$$

upon which a similar argument for an integrated mass-function as the one from (43) and (44) can be based.

Performing analogous steps as in the diagonal gauge we obtain in the limit of $\lim _{r \rightarrow \infty}$ the Bondi-mass at $\mathcal{I}^{-}$

$$
\hat{m}_{-}(v)=\lim _{r \rightarrow \infty} \hat{m}(v, r)=\hat{m}(v, \infty ; \infty, 0)=\int_{\infty}^{v} d v^{\prime} \hat{A}\left(v^{\prime}, \infty\right)+\hat{m}_{A D M}
$$

and similarly the ADM-mass

$$
\hat{m}_{A D M}=\hat{m}_{-}(\infty)=\hat{m}(\infty, \infty ; \infty, 0)=\int_{0}^{\infty} d r^{\prime} \hat{B}\left(\infty, r^{\prime}\right)+\hat{m}_{0} .
$$

Of course, a similar set of equations is obtained for asymptotically ( $h \rightarrow 1$, $(e) \rightarrow 1$ ) outgoing BS gauge

$$
(d s)^{2}=(e)(h d u+2 d r) d u
$$

instead of (50), with $h$ and (e) now being functions of $u$ and $r$ leading to the Bondi mass $m_{+}(u)$ at $\mathcal{I}^{+}$.

The simple set of equations (55) and (56) should be compared with the equations to be used when the extrinsic curvature has not been eliminated [20]. This explains the difficulty to pinpoint in that work the conservation law (63) and (64) and its ensuing integrated form as in (45) and (46). 


\section{Conclusions and Outlook}

We emphasize the usefulness of a first order formulation of spherically reduced gravity. It allows in a very easy manner to specify particular gauges from a general set of first order differential equations, involving Cartan variables and auxiliary fields, one of which can be identified with the dilaton field.

However, the central point of our argument is that the (in space and time) absolute conservation law, valid for all $2 \mathrm{~d}$ theories of gravity should be properly taken into account in treatments of selfgravitating matter because it directly produces a time dependent effective black hole mass (49).

It is instructive to compare our result with the one obtained in the seminal work of Mann [15]. There are similarities in the structure of the resulting relations, but also essential differences which allow us to cover a much wider field of applications than Mann's formula is able to do. Whereas kinetic terms from scalars (and also for fermions), to be used in computer simulations as e.g. in [16], 20] fit perfectly in our generalized version of that conservation law, those physically important cases had to be excluded explicitly in 15$]$. Apart from that also our conservation law is obtained in a first stage by combining the e.o.m. (13), (14) for auxiliary variables $X, X^{ \pm}$in order to arrive at the relation (20) which only without matter $\left(W^{(m)}=0\right)$ would correspond to the conservation of $\mathcal{C}^{(g)}$ which essentially coincides with the ADM-mass. Then, as in [15] by using in addition the "genuine" e.o.m. from the Einstein-Hilbert action with matter (eqs. (15)-(17) in our paper), one can prove the integrability condition $d W^{(m)}=0$ and thus a conservation for the sum of $\mathcal{C}^{(g)}$ and $\mathcal{C}^{(m)}$.

In fact, this "two-stage" structure of the conservation law is reflected in the associated Noether-symmetry [13]. Again a formulation in terms of Cartan variables has turned out to be most illuminating. Whereas in the matterless case the Noether-symmetry reduces to the well-known transformations in the direction of the Killing-field (called $\delta \gamma$ in the second reference [13]), in the presence of matter the integrability condition $d W^{(m)}=0$, referred to above, must be interpreted as "another" conservation law for a 1-form "current" $W^{(m)}$ with associated matter-related symmetry parameters $(\delta \rho$ in the second reference [13]) which are different from the $\delta \gamma$.

\footnotetext{
${ }^{3} \mathrm{Cf}$. the remark after eq. (4) of ref 15$]$.

${ }^{4} \mathrm{Cf}$. the second reference [13]. Of course, the validity of $d W^{(m)}=0$ also follows trivially from the structure of (20).
} 
In the matterless case there is even a deeper reason for the existence of the conserved quantity: Since the geometric part of the Lagrangian (5) is a special case of a Poisson- $\sigma$ model [3], [4], one can use so-called Casimir-Darboux coordinates in order to derive the (geometric part of the) conservation law (20). For the reader not so familiar with Poisson- $\sigma$ models and its ensuing relation to first order gravity we refer to [6].

In our present paper we discuss the conservation law including matter for the diagonal gauge [16, 18] and for the Bondi-Sachs gauge [19].

Comparing for the diagonal gauge the equations used for numerical simulations for black hole formation we find that the conservation law is intimately related to a well-known differential equation of the time derivative of the mass aspect function $m(t, r)$ in the diagonal gauge. The conservation law for $m=m(t, r)$ is formulated in terms of an integral over the scalar fields and an additional free constant $m_{0}$ which may be interpreted as the initial value of the geometric part of the action. The latter, together with the initial value of the scalar field through (47) determines the solution and is identical with the ADM mass. The mass aspect function at $i_{0}$, eq. (49), represents the effective black hole mass at a certain time $t$. It depends on the in- and outgoing matter fluxes thus leading to a Bondi-like mass definition already in a diagonal gauge.

In the Bondi-Sachs gauge we obtain a remarkably simple relationship between the Bondi mass, the ADM mass, the conservation law and the mass aspect function, summarized in eqs. (65) and (66). Also, the other e.o.m.'s are much simpler than the ones in some recent literature [20, since we are able to avoid altogether the introduction of the extrinsic curvature as a dynamical variable.

Numerous further applications of our present approach are obvious:

Spherically reduced Einstein-gravity in $d>4$ dimensions in the line element (1) only shows a different power of the dilaton field

$$
(d s)^{2}=g_{\mu \nu} d x^{\mu} d x^{\nu}-\frac{(d-2)^{\frac{4}{(d-2)}}}{\lambda^{2}} e^{-\phi \frac{4}{(d-2)}}(d \Omega)^{2}
$$

which leads to a replacement of the "potential" $\mathcal{V}$ in the geometric part of

\footnotetext{
${ }^{5}$ The factor of $(d \Omega)^{2}$ has been chosen such that the dilaton field is dimensionless and in the $\operatorname{limit}_{d \rightarrow \infty} \lim _{d \rightarrow \infty}$ we obtain the CGHS model (c.f. fourth reference of [8]) for some finite $\lambda$ of mass dimension one.
} 
the action (5) by

$$
\mathcal{V}^{(d)}=-\frac{(d-3)}{(d-2)} \frac{X^{+} X^{-}}{X}-\frac{\lambda^{2}}{2} \frac{(d-3)}{(d-2)} X^{\frac{d-4}{d-2}} .
$$

The integrating factor in the conservation law changes from $X^{-\frac{1}{2}}$ to $X^{-\frac{d-3}{d-2}}$. With the gauge fixing $X=\frac{(\lambda r)^{(d-2)}}{4}$ all further steps are exactly as in the case $d=4$.

The conservation law (23), of course, also appears when other types of matter are considered. In conformal gauge (cf. e.g. [27]) for a $2 \mathrm{~d}$ line element $(d s)^{2}=2 e^{2 \rho} d u d v$ the proper gauge in (1) is $e_{u}^{-}=e_{v}^{+}=0, e_{u}^{+}=e_{v}^{-}=e^{\rho}$.

Other gauges may be chosen with equal ease. For instance the area $A(t, r)=8 \pi X / \lambda^{2}$ of the surface $S^{2}$ could be retained as an independent variable, and, say, in $g_{\mu \nu}$ the BS gauge could be restricted further by requiring the $2 \mathrm{~d}$ volume $\operatorname{det}(e)=1$ as in the Schwarzschild gauge.

So far only spherically reduced Einstein gravity has been considered. The generalization to the Einstein deSitter case is obvious as well. A nonvanishing cosmological constant $\Lambda$ simply changes the "potential" $\mathcal{V}$ to

$$
\mathcal{V}_{\text {deSitter }}^{(d=4)}=-\frac{X^{+} X^{-}}{2 X}-\frac{\lambda^{2}}{4}+\Lambda X
$$

Then, e.g. in the diagonal gauge of section 3, neither the e.o.m. for (32), (33) for the scalar field, nor the conservation law equations (43), (44) are changed, if the mass aspect function is redefined as

$$
a^{-2}=1-\frac{2 m(t, r)}{r}-\frac{\Lambda r^{2}}{3} .
$$

Only the "slicing-condition" (34) acquires an additional term

$$
\frac{\partial}{\partial r} \ln \left(\frac{\alpha}{a}\right)+\frac{1-a^{2}}{r}+\frac{\Lambda a^{2} r}{2}=0
$$

as well as the "Hamiltonian constraint" (34)

$$
\frac{\partial}{\partial r} \ln a+\frac{a^{2}-1}{2 r}-\frac{\Lambda a^{2} r}{2}=2 \pi r\left(\Phi^{2}+\Pi^{2}\right) .
$$

Different types of matter can be discussed either by inserting the appropriate (spherically reduced) matter action or, more directly, from the corresponding (spherically reduced) energy momentum tensor $T^{\mu \nu}$. Then instead 
of the $S$-dependent terms the matter interaction in (14) is proportional to (e) $\varepsilon_{\rho \mu} T^{\rho \sigma} e_{\sigma}^{ \pm}$. This would be needed e.g. for the case of a perfect fluid which also has been studied in connection with black hole formation [28]. One of the main advantages of the $2 \mathrm{~d}$ spherically reduced gravity formulation is the ease by which a transition from one $2 \mathrm{~d}$ gauge to another is possible and the ease by which the absolute conservation law (23) can be derived. We emphasize once again that the appearance of the latter is peculiar to $d=2$ (or to a $2 \mathrm{~d}$ reduction of a higher dimensional theory).

Finally it should be mentioned that the conservation law in the presence of matter is related to a Noether-symmetry of a somewhat unusual type [13. This (nonlinear) symmetry so far is known only in infinitesimal form. Possibly further investigations in that direction could also contribute towards the understanding of the intriguing results from numerical studies of spherical black hole formation.

\section{Acknowledgements}

The authors have benefitted from discussions with H. Balasin, D. Hofmann, W. Waltenberger and R. Wimmer. They thank M. W. Choptuik for cor-

respondence. This research has been supported by Project P12815-TPH of the Austrian Science Foundation (Österreichischer Fonds zur Förderung der wissenschaftlichen Forschung).

\section{References}

[1] W. Kummer and D. Schwarz, Phys. Rev. D 45 (1992) 3628.

[2] N. Ikeda and K-I. Izawa, Progr. Theor. Phys. 89 (1993) 1.

[3] T. Strobl, Phys. Rev. D 50 (1994) 7346.

[4] P. Schaller and T. Strobl, Mod. Phys. Lett. A 9 (1994) 3129.

[5] W. Kummer and P. Widerin, Phys. Rev. D 52 (1995) 6965.

[6] T. Klösch and T. Strobl, Class. Quant. Grav. 13 (1996) 965; Corr. ibid. 14 (1997) 825. 
[7] D. Louis-Martinez, J. Gegenberg and G. Kunstatter, Phys. Lett. B 321 (1994), 193; Phys. Rev. D 51 (1995), 1781; W. M. Seiler and R.W. Tucker, Phys. Rev. D 53 (1996), 4366; A. Barvinsky and G. Kunstatter, Phys. Lett. B 389 (1996), 231.

[8] G. Mandal, A. Sengupta and S. R. Wadia, Mod. Phys. Lett. A 6, (1991), 1685; S. Elitzur, A. Forge and E. Rabinovici, Nucl. Phys. B 359 (1991), 581; E. Witten, Phys. Rev. D 44 (1991), 314; C. G. Callan, S. B. Giddings, J. A. Harvey, and A. Strominger, Phys. Rev. D 45, (1992), 1005; J. Russo, L. Susskind and L. Thorlacius, Phys. Lett. B 292 (1992), 13.

[9] T. Klösch and T. Strobl, Class. Quantum. Grav. 13 (1996) 2395.

[10] M. O. Katanaev, J. Math. Phys. 34 (1993) 700.

[11] M. O. Katanaev and I. V. Volovich, Phys. Lett. B 175 (1986) 413.

[12] W. Kummer and D.J. Schwarz, Nucl. Phys. B 382 (1992) 171; F. Haider and W. Kummer, Int. Journ. Mod. Phys. A 9 (1994) 207; W. Kummer, H. Liebl and D. V. Vassilevich, Nucl. Phys. B 493 (1997) 491 and B 513 (1998) 723; W. Kummer, H. Liebl and D. V. Vassilevich, Nucl. Phys. B 544 (1999) 403.

[13] W. Kummer and P. Widerin, Mod. Phys. Lett. A 9 (1994) 1407; W. Kummer and G. Tieber, Phys. Rev. D 59 (1999) 044001.

[14] T. Banks and M. O'Loughlin, Nucl. Phys. B 362 (1991) 649.

[15] R. B. Mann, Phys. Rev. D 47 (1993) 4438.

[16] M. W. Choptuik, PHD-thesis, Univ. of British Columbia 1986; Phys. Rev. Lett. 70 (1993) 9.

[17] D. Goldwirth and T. Piran, Phys. Rev. D 36 (1987) 3575.

[18] C. Gundlach, Adv. Theor. Math. Phys. 2 (1998) 1; M. W. Choptuik, The (unstable) threshold of black hole formation, gr-qc/9803075.

[19] R. K. Sachs, Relativity, groups and topology, ed. C. de Witt and B. S. de Witt (1964), New York: Gordon and Breach.

[20] R. L. Marsa, M. W. Choptuik, Phys. Rev. D 54 (1996) 4929. 
[21] P. Thomi, B. Isaak and P. Hajicek, Phys. Rev. D 30 (1984) 1168; P. Hajicek, ibid. D 30 (1984) 1178; S.R. Lau, Class. Quant. Grav. 13 (1996) 1541.

[22] M. O. Katanaev, W. Kummer and H. Liebl, Phys. Rev. D 53 (1996) 5609; W. Kummer, H. Liebl and D. V. Vassilevich, Nucl. Phys. B 493 (1997) 491.

[23] M. O. Katanaev, T. Klösch and W. Kummer, Global properties of warped solutions in general relativity, gr-qc/9807079 (to be published in Ann. Phys. (N.Y.)).

[24] V. Mukhanov, A. Wipf and A. Zelnikov, Phys. Lett. B 332 (1994) 238; W. Kummer and D. V. Vassilevich, Phys. Rev. D 60 (1999) 084021; V. Frolov, P. Sutton, A. Zelnikov, The Dimensional-Reduction Anomaly, hep-th/9909086 v2.

[25] R. Arnowitt, S. Deser and C. W. Misner, The Dynamics of General Relativity in Gravitation: An Introduction to Current Research, ed. L. Witten (1962) New York: Wiley.

[26] D. Christodoulou, Comm. Math. Phys. 93 (1984) 171; 106 (1986) 587; 109 (1987) 613

[27] A. V. Frolov, Phys. Rev. D 56 (1997) 6433.

[28] T. Koike, T. Harara and S. Adachi, Phys. Rev. Lett. 74 (1995) 5170; D. Maison, Phys. Lett. B 366 (1996) 82. 